\title{
Educations Board Game for Sustainable Agriculture - Pheromone Pest Control
}

\author{
Wen-Chih Chou ${ }^{1}$, Wei-Zhong Feng ${ }^{2} \&$ Hui-Ping Liu ${ }^{1}$ \\ ${ }^{1}$ Department of Industrial Design, Chaoyang University of Technology, Taichung, 436, Taiwan \\ ${ }^{2}$ Department of Visual Communication, Chaoyang University of Technology, Taichung, 436, Taiwan \\ Correspondence: Wei-Zhong Feng, Department of Visual Communication, Chaoyang University of Technology, \\ Taichung, 436, Taiwan.
}

Received: August 23, 2021

Accepted: September 22, 2021

Online Published: October 7, 2021

doi:10.20849/jess.v4i1.936

URL: https://doi.org/10.20849/jess.v4i1.936

\begin{abstract}
The mode of agricultural operation affects the ecological environment, and the pollution of land by chemical pesticides also affects the environmental balance. The death of many bees affects pollination, and the animals in the upper layers of the food chain die. The pheromones are formulated with synthetic pheromones as bait and then trapped with appropriate insect traps to reduce the chance of pest mating. Table game matches the props of the garden and pests by moving the insects to the traps. The model constructed by the relationship between the shape of the bottom of the insect and the track. Augmented reality provides an interactive experience of an event-driven where the objects reside in the real world with computer-generated information. Users can use the QR codes to trigger online media. When students complete a specific task, they scan the QR Code with their mobile phone and get more exciting instructions and task guidance. Students satisfied to more in-depth knowledge of pheromones with AR too.
\end{abstract}

Keywords: educations board game, sustainable agriculture, pheromone pest control, augmented reality

\section{Introduction}

\subsection{Why the Issue Investigated Is an Issue of Interest}

Pheromone's technology has been used in pest control, and it is gradually spreading to different crops. But the problem encountered by promoters is that the people who use these technologies are farmers, and they do not have scientific knowledge, which affects their willingness to accept. Therefore, education is needed to let them understand the principles of Pheromone and how they are different from the use of traditional pesticides. Then they are willing to use them in their workplaces. Formal agricultural education has no interactive fun, so through games and other relatively easy ways to bring this group to let. Relatives trigger the interest in learning in the game, and the acceptance of such technical principles also raises the awareness of environmental protection.

\subsection{The Approaches in This Study}

First, we will explore the types of pests on the main agricultural fields in Taiwan and preliminarily classify them into four major items. In response to the abstract characteristics of this worm body, we designed a pattern that children can recognize. When children find similar pests in the field, they can tell which Pheromone should be selected to achieve the desired purpose. Secondly, we design a competitive game method so that the two parties can generate a sense of accomplishment through competition. Finally, we also use to understand the level of acceptance among farmers, adjust the interpretive information and apply it in environmental education.

\subsection{Sustainable Agriculture}

World Health Organization (2015) claimed the climate change and over-exploitation which caused the change of eco-system. In 2015, the United Nations listed sustainable agriculture as an indicator in the Sustainable Development Goals. The Millennium Development Goals (MDGs) come to a post-2015 agenda, comprising 17 Sustainable Development Goals for the new era. Sustainable agriculture refers to reducing environmental impacts from production, harvesting, processing to market entry. The agricultural waste caused air pollution, soil pollution caused by pesticides, heavy metals, and veterinary drugs; water pollution caused by pesticides and feces. 
The excessive use of pesticides and nitrogenous fertilizers affects shallow groundwater and freshwater quality (Rasul 2016). Based on the principle of "reduction, reuse, and resource utilization," the government hopes to form a development model with low consumption, low emissions, and high efficiency. Sustainable agriculture has been a concern due to the implications for food safety, a goal of zero growth set to make agriculture sustainable (Shuqin and Zhou 2018).

\subsection{Environmental Education}

The Legislative Yuan of Taiwan passed the Environmental Education Act in 2011 (Ho 2017). This law makes people are more environmentally conscious at school and society. In Article 19 of the Environmental Education Act, the EPA of the Executive Yuan requested schools select facilities and sites for conducting environmental education. Attractive education facilities have a demand by adults and students. Board game is welcome by youth, therefore this design could encourage students to learn sustainable problems through playing.

The collaborative and interdisciplinary teaching curriculum was developed for students and community partners (Culhane 2016). Clean production used green management to promote recycling agriculture. From the source process control, the agricultural resources supervision information and traceability platform for safety fertilizers and pesticides.

Theoretical frameworks developed on the possession of knowledge and awareness (Stapp 1969). Factors included demographic, institutional, economic, and cultural factors. Knowledge and experiences have an inference of people's behavior. People with pro-environmental attitudes are more likely to engage in protective acts. The game enables people to experience situations. The educational game extends the knowledge domain and brings the exploratory attempt to related knowledge.

\section{Pheromone Pest Control}

Some farmers have begun to use "friendly farming" to maintain water and soil resources, biodiversity, and promote environmental sustainability. "Pheromone Control" is a method of controlling pests that have been supported in recent years (Kydonieus 2019). The "Reproductive Interference Method" is to curb biological mating and achieve the effect of controlling pests. The pheromone molecule is released from the gland and diffuses in a spherical state to form a concentration gradient. The male is attracted to the pheromones in the trap and enters the trap from the upper hole (Figure 1). Because of the individual entrance and non-reversal bottle design, it is impossible to fly out after entering (Lin and Hsieh 2018). A micro-particle structure for a slow release of an insect attractant pheromone includes a core and a shell layer. The core layer may consist of a carrier and a first attractant pheromone, wherein the carrier is formed of a biodegradable material. The microparticle structure (Figure 2) release of an insect attractant pheromone of the present disclosure is applied to the soil 300, thereby releasing the second attractant pheromone 220 and degrading enzyme and the fertilizer 130 within the core layer.

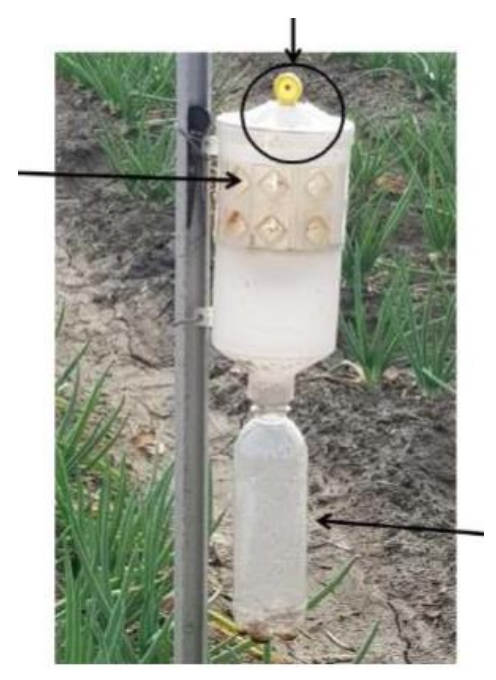

Figure 1. Insect trap structure, where left- insect enter hole, right- a dead body, upper circle- pheromone attached zone 


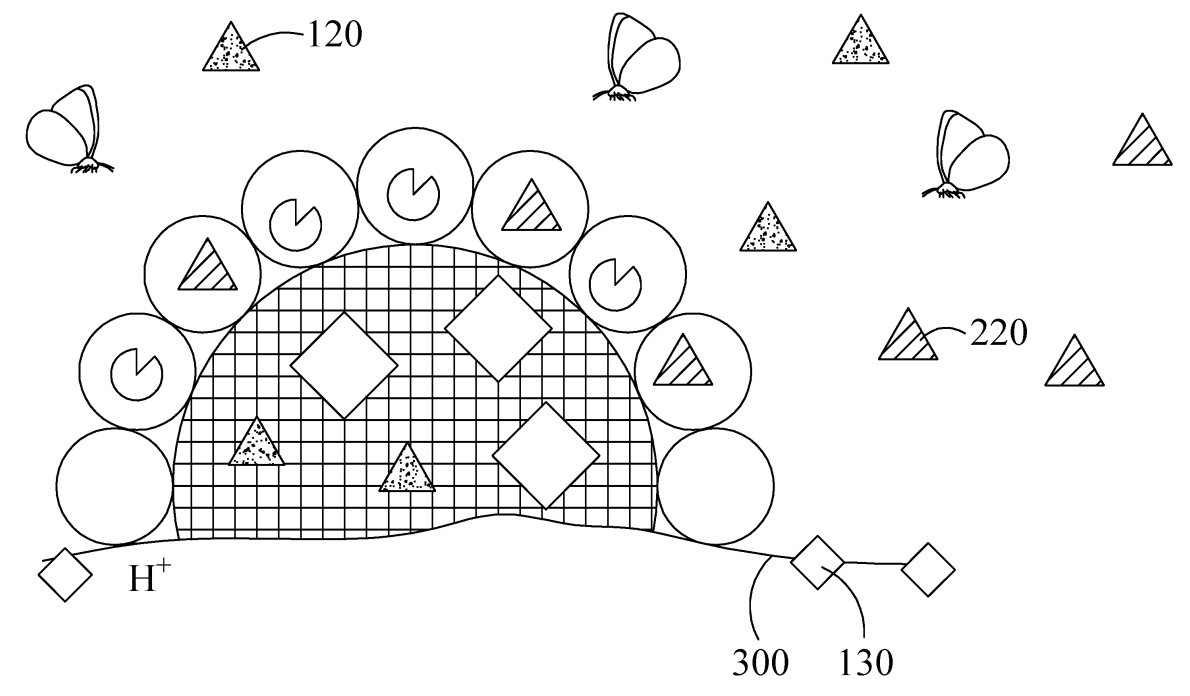

Figure 2. Schematic diagram, where 300-soil, 220-attractant pheromone, 110-the carrier, and the 130-fertilizer

Previous studies demonstrated that extracts from EPN-infected hosts enhance dispersal and efficacy (Oliveira-Hofman and Shapiro-Ilan 2019). Some active components in the insect host cadavers responsible for dispersal, ascarosides, have been identified as nematode pheromones. "Pheromone extracts induced higher numbers of Steinernema." The high concentration of pheromones in the field can hinder the normal mating of the females and males and inhibit the occurrence of their ethnic groups. Sex pheromone dosages and release point densities for mating disruption shows positive results (Chen 2017). Pheromones to control Taiwan's field pests are:

(1) Spodoptera litura:

The twill night thief is an omnivorous pest that can be found throughout the year. During the day, it hides under the residual leaves of the base of the plant and goes out to feed at night, and the leaves are eaten to endanger the tender stems.

(2) Chitosan:

Dive into the inside of the rice sheath. Adults have strong phototaxis for nocturnal behavior, and prevention and control should carry out before they harmed into the stem.

(3) Plutella xylostella:

It is the leading pest of cruciferous crops. The eggs laid along the veins, and the hatchets will sneak into the leaves after hatching. The flower parts, fruit pods, and stem epidermis also affected.

(4) Sweet potato ant elephant:

Adults like to inhabit in the dark or sweet potato leaf vines, the traces of the damaged sweet potato are brown and corked, and smelly.

\section{Board Game Design}

\subsection{Concept}

The board games are popular in the youth group (Figure 3). The Tourism Bureau will create a Taiwanese folklore feature "Swordsman" as a board game. In conjunction with the 2018 Taiwan Lantern Festival in Chiayi, the picture book "Jianshi Lantern Festival" will be launched. In the early decorations of many temples and ancient houses, the pottery sword lions, which can avoid evil, stood high on the door wall to protect the residents from entering and exiting safely. The board game initiated by the sword lion's "sword is missing." Participants were able to help the anxious lion follow the clues to find the place of the lantern festival and the bear brother who took the sword.

We simulate the garden situation and let people experience the principles of pheromones. Learn the pest control 
knowledge by moving the insects to the game in the trap. Pheromone control is bait with a suitable insect trap to reduce the chance of pest mating. The principle of pheromones is recognized by the shape of the bottom and the track. When people are curious, they will find the principles behind (Liu 2019). The core layer has a carrier, an attractant pheromone, and a carrier with biodegradable material. The shell includes many microcapsules, chitosan shell with pheromone.

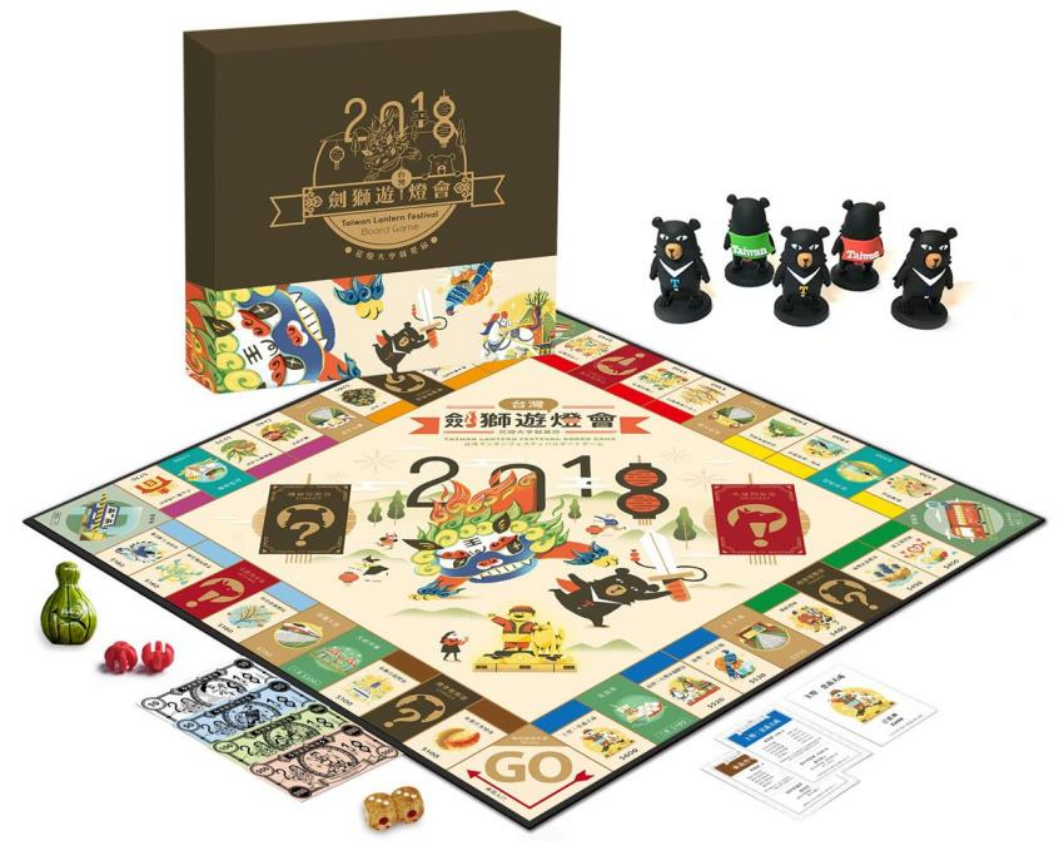

Figure 3. Taiwan lantern board game: Sword lion tour lantern festival

\begin{abstract}
"Game Experience Aids" is designed for teaching purposes and incorporates game elements in the interactive process. The "situational type" of environmental simulation is expressed in the form of a map to assist the "story context." The pheromone-inducing knowledge-based board game allows rural experiencers to buy home experiences after listening to pastoral planting tours. It is made up of 2 to 4 people and is made of wood and photosensitive resin. Insect design reveals its characters, shape, and texture. Farm field emulated farmland and the ecology. The concept sketch comparison shows the factors and scores (Figure 4). a) Bead bounce path game: The bead is bounced through the same color platform into the same color hole to express the relationship between specific insects and pheromones. b) Convert the insect and pheromone-specific relationship into a path of different materials, and combine the tracks of the same material to roll the beads into the storage box. c) Insects move through the path into the trap.

Concept selection factors include insect traps, insect objects, and expression of gas diffusion, the pairing of insects and pheromones, and interpretation of gas molecules. Different concepts represent characteristics of pheromones, but users who are in contact for the first time need to understand the most critical aspects. It is the kind of coupled microstructure, which shows the strange attractive pairing between unique species. Through interviews and evaluations, the values of the factors in Concept $\mathrm{C}$ are the highest and selected. In the detail design stage, we developed a board game design for this concept.
\end{abstract}




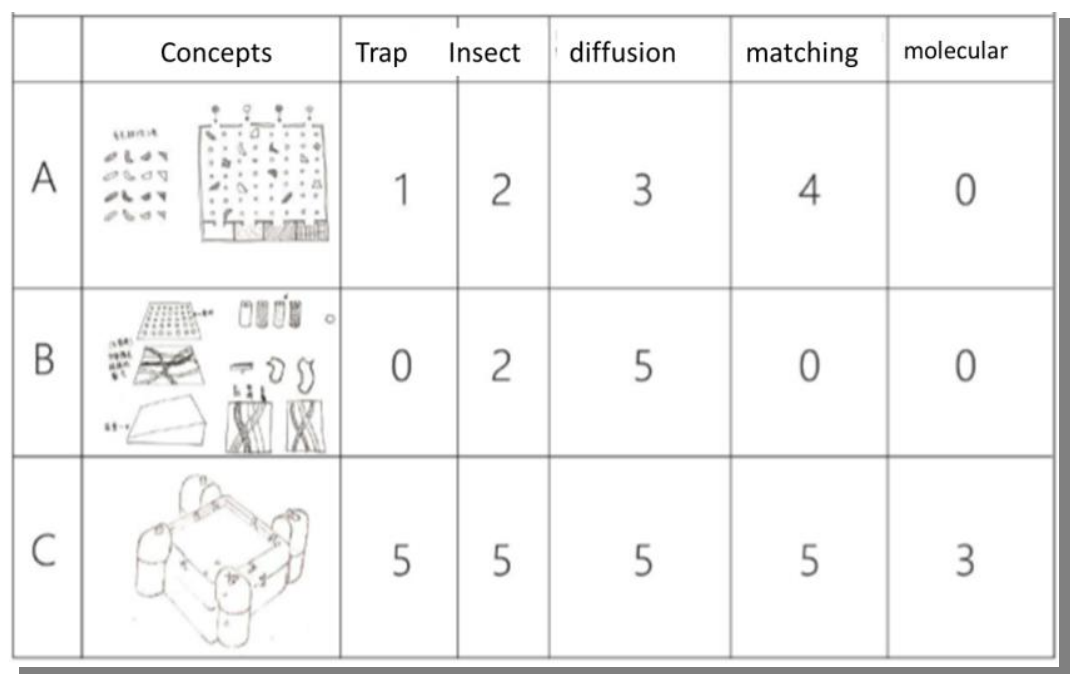

Figure 4. Concept sketch comparison: the highest score on the scale is 5 points

\subsection{Interface Design}

Rules of matches are present to facilitate communications between players. The sensation of fun may derive from mastering new knowledge, skills, or experience. Story and mechanics involve the procedure that provides the structure by which the user interacts with system elements. Users invest skills to reach the goal and get pleasure through achieving and confidence (Chawla and Cushing 2007).

While playing, users have to concentrate on current situations, and practice strategy requires reaching the goal. The board game included plate, game cards, tools, and the rules that provide information with which a person may interact. The great attraction allows people to enjoy playing. Nielsen's mentioned principles for interaction design (Nilsson 2009) as follow:

(1) Strive for consistency: users should not wonder whether different words or situations mean the same thing.

(2) Visibility of system status and offer feedback: The system should keep users informed about what is going on through appropriate feedback.

(3) Match the real world to yield closure: The system should speak the users' language.

(4) Reduce short-term memory load: recognizing something is more natural than remembering it. Minimize the user's memory load by making objects with previous experience.

Augmented reality provides an interactive experience of an event-driven where the objects reside in the real world with computer-generated information. Users can use the AR-Icon or QR codes to trigger online media, and digital content is behind it. The app supported transform a smartphone into an AR viewer. The Museum of Modern Art in New York developed an exhibit showcasing AR features that viewers can use smartphones using MoMAR Gallery app (Efrat 2020) which explicitly defining both space and art as exclusive and invulnerable. Students want to be exposed to more in-depth knowledge of pheromones, so we supplement this game with augmented reality mechanisms. When students complete a specific task, they can scan the QR Code with their mobile phone and get more exciting instructions and task guidance after triggering. The Aurasma app is popular in education; in Delello (2015), researchers examined the use of the Aurasma in the classroom. Holzschuh (2017) described application in Geography teaching with positive feedback. In Hudaya (2019), the author investigated vocabulary mastery through AR facilities.

\subsection{Detail of Design}

The 3-D board game concentrates on pheromones-related components, including board, pest dice, trees, event cards, and flags. The designer suggests a suitable age range of users for playing the game is above 10. There are four kinds of insects (6 each), 30 pieces, and a total of 4 traps. Insects can be stored or taken out in whole sets. Designing the path to an inverted cone allows the flying piece to move smoothly over the path board without falling. The Aurasma app trigger with arranged QR code in specific domain (Figure 5). The functional model was created and confirmed to stick at the turn (Figures 6,7). 


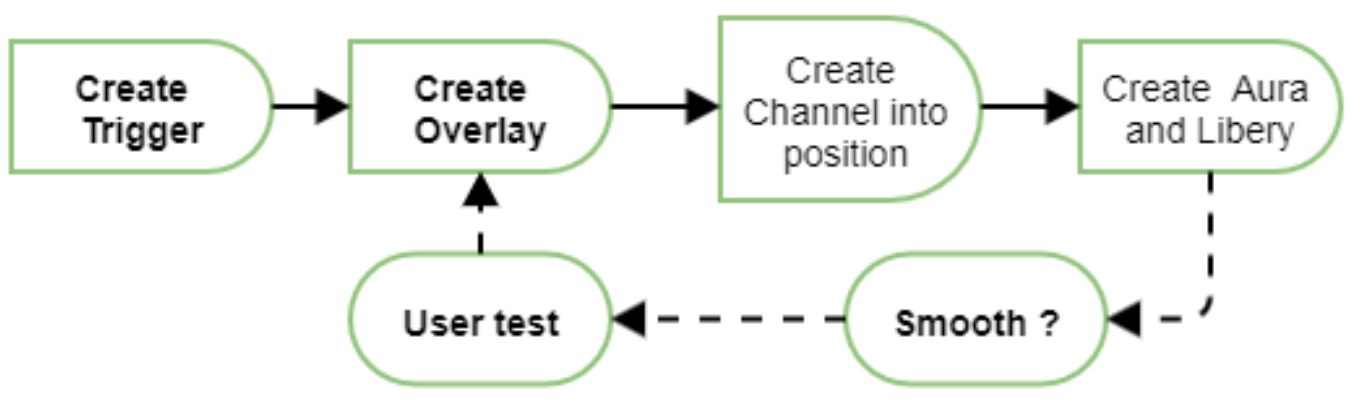

Figure 5. The Aurasma app design flow

The entrance and exit of each path are provided with protrusions such as semicircles, rectangles, and right triangles to make the path chessboard conform. There are depressions of the same shape under the insects, making it impossible for other insects to enter. The path board uses different shades of green to simulate the appearance of the garden. The presence of the tomato night moth and the diamondback moth is distinct for insect identification (Figure 8).

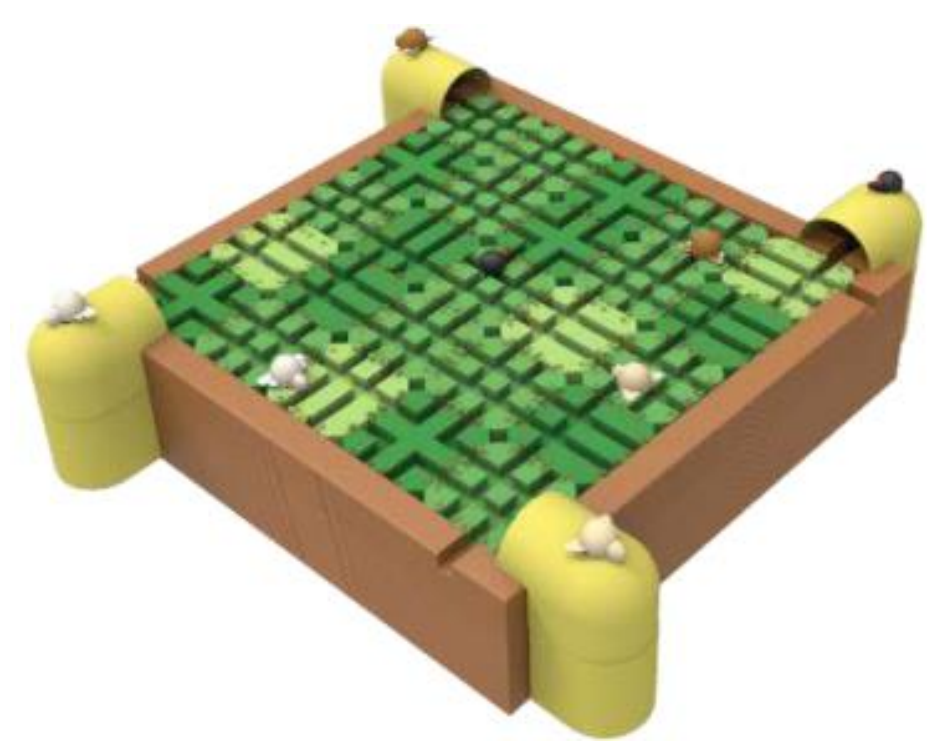

Figure 6. Pheromones board game, where the main table is a 3D structure with different paths and insects (corner tube is the trap storage)

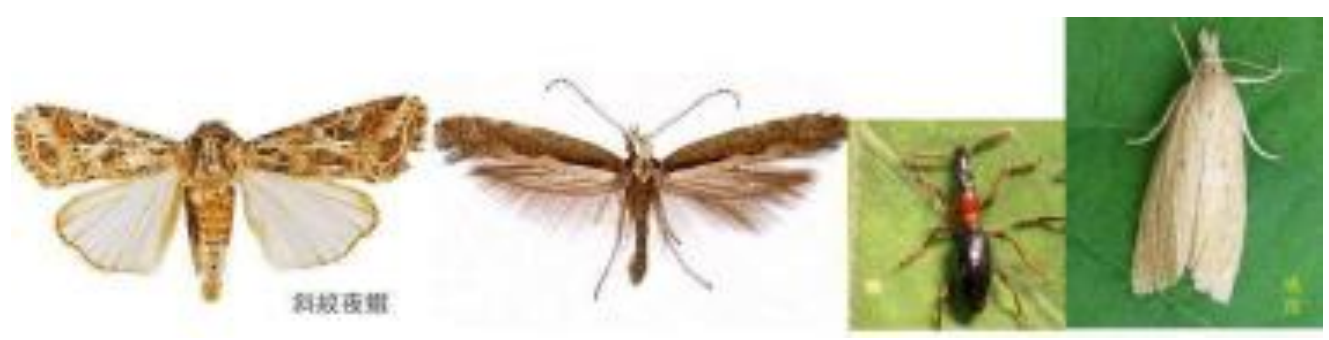

(a) 


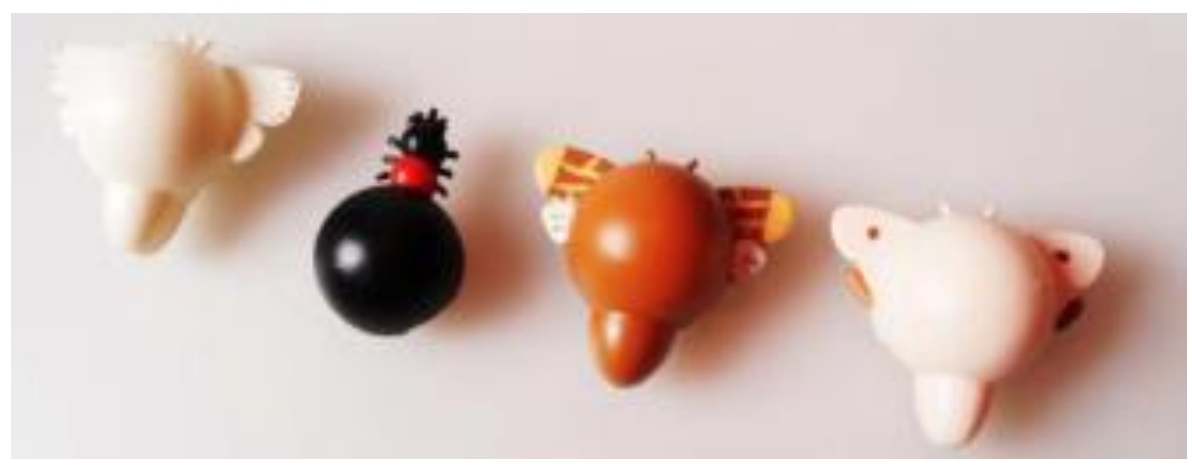

(b)

Figure 7. Comparison of insects and feature transfer (a) real object, (b) 3D printed model. Use the color of the insect with simplifying shape and texture

The designer implemented the theme and response to the situation in design. The first one moves all insect reaches the trap area is the winner. Through the contents of the event card, we bring the player into a different situation. The player can acquire strategy and trapping of insects. Each person has five pests, and the target of the trap is selected to move forward from the board. When you are blocked, you can walk or turn the board block. When you encounter obstacles, you need to bypass it.

\section{4 "Pheromone Pairing" Study Sheet}

Moths have a short life span (a few days), and to complete mating, they must court couples very efficiently. How did they date and match?

The chemical substance released by the female abdomen to lure males to mate is sexual pheromones. Sexual pheromone gland protrudes from the abdomen during release, showing courtship behavior. Its chemical structure differs depending on the type of insect and is composed of several compounds mixed in a specific ratio (different ratios) so that individuals with the same particular species can mate. The sex pheromones of corn borer are composed of isomer $\mathrm{Z}$ and isomer $\mathrm{E}$ with a ratio of 97:3, but the rate of $\mathrm{E}$ corn borer is 99:1, which plays a role in reproductive isolation to avoid mating between different types of insects.

\section{Evaluation}

While the prototype constructed, the elderly (55 65 years old) of different age groups are invited to play and gather user response. After participants accepted 5 minutes background introduction and read the "Study Sheet," the pair of users engaged together in 20 mins playing session (Figure 8). Firstly, the four elders put their insects into the trap. In the beginning, the four elders were not familiar with the rules, so they explained while operating. After three steps and familiar with the game, they know how to plan the walking route. After the test, the researcher asked four subjects about the game and suggestions for improvement. The evaluator recorded a log of the player's actions and failures and coded each of these within observation record. Most of them have positive feedback.

(1) The game was easy to use and new experience with AR.

(2) The path color avoid the inability to distinguish.

Insect doll and track groove are attractive.

(3) Through the shape of the track grooves of different kinds of insects in the board game, AR supported chemical backgrounds.

(4) Some terminologies are not familiar to them, so the elderly acquired additional explanation.

(5) Being lacking the beginning level of the knowledge, a player did not have experience of judging. The AR script is helpful with the scenarios. 


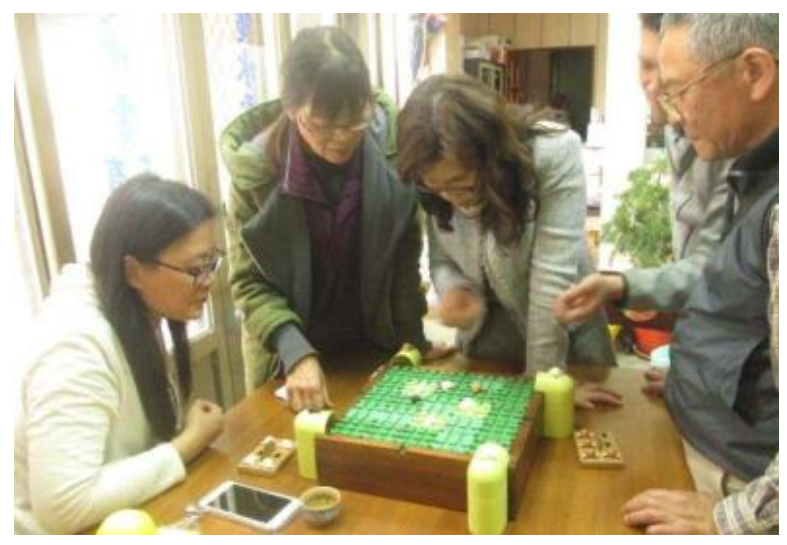

Figure 8. Prototype evaluation

\section{Conclusion}

The storytelling and analogy structure of the pheromone was used in the game. Simplify the terminology reduces the complexity of its association. Through observation records, most of them have positive feedback. After playing the board game, the elderly are willing to understand more about how things are happening in our surroundings. Through the shape of the track grooves of different kinds of insects in the board game, participants experienced the chemical principle that pheromones need to be paired with each other. Users can use the QR codes to trigger online media. When users complete a specific task, they scan the QR Code with their mobile phone and get more exciting instructions and task guidance; they satisfied AR experience with in-depth knowledge.

The limits of this research are the abstract characteristics of this worm body. This design only shows the difference in the morphology of the final door pairing. There are some changes in the diffusion mechanism and the method of attracting insects; this need to present in future designs. However, we have also verified that these game boards and graphics cards are connected with actual agricultural field knowledge, which can activate farmers' interest in learning. In the future, by further expanding the link content through VR, they can further develop their knowledge acceptance during their play. Although VR technology makes people feel immersive, it has learning barriers or a sense of rejection for farmers. Therefore, we use a simple QR Code to allow them to connect. In the future, we need to further introduce the technology according to the farmers' abilities and specific concerns.

\section{References}

Chawla, L., \& Cushing, D. F. (2007). Education for strategic environmental behavior. Environmental Education Research, 13(4), 437-452.

Chen, R. Z., Jow, C. K., Klein, M. G., Jia, Y. D., Zhang, D. Y., \& Li, L. B. (2017). Sex Pheromone dosages and release point densities for mating disruption of ostrinia furnacalis (Lepidoptera: Crambidae) in NE China corn fields. Environmental Entomology, 46(4), 916-925.

Culhane, J. H., et al.. (2016). Learning through collaborative and interdisciplinary teaching: a case study of faculty work as learning in sustainable agriculture education. NACTA Journal, 60(2).

Delello, J. A., Rochell, R., McWhoRte, R., \& Kerri, M. C. (2015). Integrating augmented reality in higher education: A multidisciplinary study of student perceptions. Journal of Educational Multimedia and Hypermedia, 24(3), 209-233.

Efrat, L. (2020). REALational perspectives: strategies for expanding beyond the here and now in mobile augmented reality (AR) art. Leonardo, 53(4), 374-379.

Ho, S. Y., Chen, W. T., \& Hsu, W. L. (2017). Assessment system for junior high schools in Taiwan to select environmental education facilities and sites. EURASIA Journal of Mathematics, Science \& Technology Education, 13(5), 1485-1499.

Holzschuh, V. R. B., \& Bogoni, T. N. (2017). Aurasma: A Tool for Education. 19th Symposium on Virtual and Augmented Reality (SVR). IEEE.

Hudaya, A. (2019). The effect of aurasma augmented reality to enhance young learners' vocabulary mastery. 
Professional Journal of English Education, 2(6), 783-790.

Kydonieus, A. F. (2019). Insect suppression with controlled-release pheromone systems. (vol. 1). CRC Press.

Lin, M. C., Chien, W-J., Yang, C-C., Shen, Y-J., \& Hsieh, W. T. (2018). Microparticle Structure for Slow Release of Insect Attractant Pheromone. U.S. Patent Application 15/643,118, filed January 25.

Liu, H. (2019). Friendly agricultural education teaching aid design. Design report, Chaoyang University of Technology.

Nilsson, E. G. (2009). Design patterns for user interface for mobile applications. Advances in Engineering Software, 40(12), 1318-1328.

Oliveira-Hofman, C., Kaplan, F., Stevens, G., Lewis, E., Wu, S., Alborn, H. T., \& Shapiro-Ilan, D. I. (2019). Pheromone extracts act as boosters for entomopathogenic nematodes efficacy. Journal of invertebrate pathology, 164, 38-42.

Rasul, G. (2016). Managing the food, water, and energy nexus for achieving the sustainable development goals in south Asia. Environmental Development, 18, 14-25.

Shuqin, J., \& Zhou, F. (2018). Zero growth of chemical fertilizer and pesticide use: China's objectives, progress, and challenges. Journal of resources and ecology, 9(1), 50-59.

Stapp, W. B. (1969). The concept of environmental education. Environmental Education, 1(1), 30.

World Health Organization. (2015). Health in 2015: from MDGs, millennium development goals to SDGs, sustainable development goals.

\section{Copyrights}

Copyright for this article is retained by the author(s), with first publication rights granted to the journal.

This is an open-access article distributed under the terms and conditions of the Creative Commons Attribution license (http://creativecommons.org/licenses/by/4.0/). 